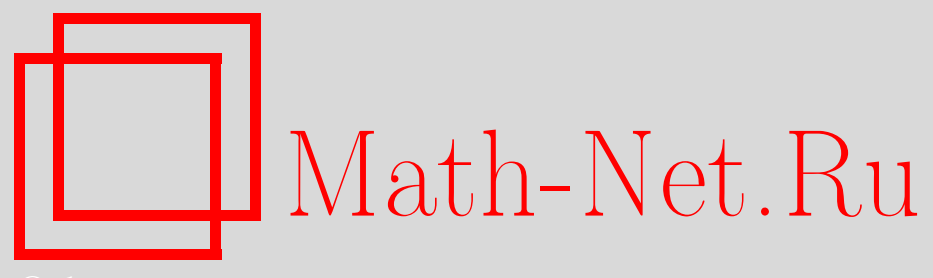

Л. Ашкинази, Почему обжигает пар?, Квант, 2020, номер 9, 33-35

DOI: https://doi.org/10.4213/kvant20200905

Использование Общероссийского математического портала Math-Net.Ru подразумевает, что вы прочитали и согласны с пользовательским соглашением http://www.mathnet.ru/rus/agreement

Параметры загрузки:

IP : 54.89 .56 .158

26 апреля 2023 г., $16: 13: 07$

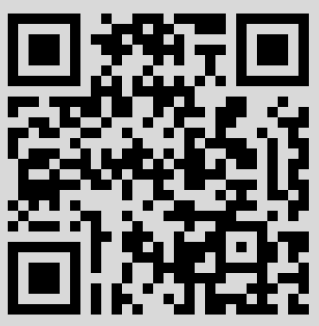




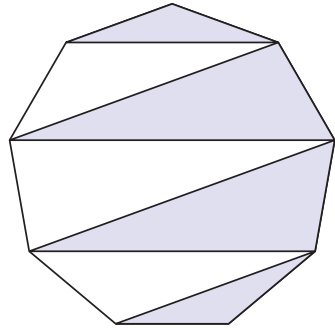

Рис. 13

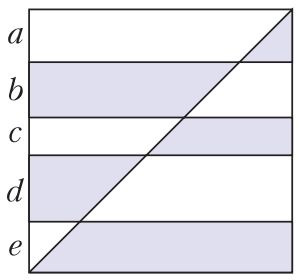

Рис. 14

(рис.13). Какая часть площади больше: белая или закрашенная?

19. Квадрат разрезан на полоски, ширина которых обозначена буквами $a, b, c, d, e$, проведена его диагональ и полученные части закрашены так, как показано на рисунке 14. Известно, что $a+c+e=b+d$. Докажите, что равны суммы площадей закрашенных частей, расположенных выше и ниже диагонали.

20. Прямоугольники $A B C D$ и $K L M N$ с соответственно параллельными сторонами расположены так, как показано на рисунке 15. Докажите равенство площадей четырехугольников $A L C N$ и KBMD.

21. На стороне $A B$ параллелограмма $A B C D$ отмечена точка $P$. Постройте параллелограмм с вершиной $P$, вписанный в $A B C D$ так, что его

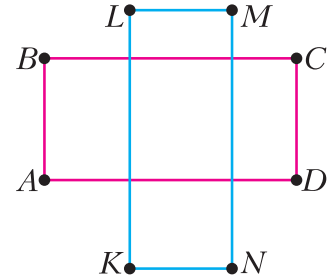

Рис. 15

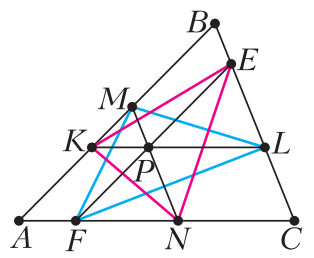

Pис. 16

стороны отсекают от исходного параллелограмма треугольники равной площади.

22. Через точку $P$ проведены отрезки $E F, K L$ и $M N$, параллельные сторонам треугольника $A B C$ (рис.16). Докажите равновеликость треугольников $K E N$ и $M L F$.

23. В треугольник $A B C$ вписана окружность с центром $I$, которая касается его сторон $B C, C A$ и $A B$ в точках $A_{1}, B_{1}$ и $C_{1}$ соответственно. Отрезки $A I, B I$ и $C I$ пересекают окружность в точках $A_{2}$, $B_{2}$ и $C_{2}$. Докажите, что площадь треугольника $A_{2} B_{2} C_{2}$ равна половине площади шестиугольника $B_{1} A_{2} C_{1} B_{2} A_{1} C_{2}$.

24. В выпуклом четырехугольнике провели отрезки, соединяющие середины противоположных сторон. Их точку пересечения соединили с вершинами четырехугольника. Докажите, что суммы площадей противолежащих треугольников равны.

\section{Почему обжигает пар?}

\section{Л.АШКИНАЗИ}

\begin{abstract}
$\mathrm{B}$ О МНОГИХ ЗАДАЧНИКАХ МОЖно встретить вопрос - «почему обжигает пар» или «почему пар обжигает сильнее, чем вода той же температуры». И дается ответ - «потому, что происходит конденсация с тепловыделением». Но, во-первых, не всякое тепловыделение заметно поднимает температуру; нужно хотя бы простейшей оценкой показать, что эффект заметен. Вовторых, вдумчивый школьник может выдви-
\end{abstract}

DOI: https://doi.org/10.4213/kvant20200905 нуть такое возражение - горячий металл обжигает сильнее горячего дерева, а холодный металл «холодит» сильнее камня, т.е. тепловые потоки зависят от теплопроводности. Пар - это газ, его теплопроводность много меньше, чем у жидкости, так что все должно быть наоборот.

Теплопроводность пара действительно мала, однако в целом это возражение неправильное. В этом нам еще предстоит разобраться, а начнем мы с понятия теплопроводности, которое употребляем интуитивно. Мы введем его для частного случая, но применяется оно шире; при этом из нашего частного вывода станет понятно ограничение, при котором можно пользоваться этим понятием.

Запишем формулу

$$
Q=c m \Delta T,
$$

которая вам известна. Здесь при чтении надо сделать короткую паузу - вспомнить, что как обозначается, написать на бумажке раз- 
мерности и убедиться, что все правильно. Попутно вопрос: позволяет ли проверка размерностей убедиться в полной правильности формулы? Нет, в формуле может быть безразмерный коэффициент - он не влияет на размерность. В данной формуле его нет, как нет его в законе Ньютона или в законе Ома. А в законе всемирного тяготения он есть, причем размерный, в законе Кулона есть и размерный и безразмерный коэффициенты - почему? Подумайте об этом на досуге.

Кроме того, вам известно понятие потока тепла, т.е. тепловой мощности, которое вы, скорее всего, определяете так:

$$
N=\frac{\Delta Q}{\Delta t},
$$

где $t$ - естественно, время. Заметим, что одни и те же, по сути, величины в разных разделах физики могут называться по-разному. Так, в электричестве $Q$ - это заряд, а $N$ - ток. Когда определение дается с «дельтами», например как здесь $N=\Delta Q / \Delta t$ или как в электричестве $I=\Delta Q / \Delta t$, подразумевается, что $\Delta t$ можно брать любое. Иными словами, $\Delta Q$ пропорционально $\Delta t$, и, какое бы $\Delta t$ мы не брали, отношение получится то же. Поэтому это определение уже предполагает, что мощность (или, соответственно, ток), постоянны. Иначе надо было бы писать $N=d Q / d t, I=d Q / d t$, дифференцировать, вводить понятия мгновенной мощности и мгновенного значения тока. Но в школе про переменное $N$ вообще не упоминается, а определение тока дается именно для постоянного тока. Однако это формальные вещи, а вот теперь нам потребуется серьезное физическое предположение.

Рассмотрим прямой цилиндр длиной $L$ и сечением $S$. Пусть на торцах цилиндра температуры постоянны, разность температур между торцами равна $\Delta T$, а боковые поверхности теплоизолированы, т.е. поток тепла через них равен нулю. Пусть в этих условиях поток тепла вдоль цилиндра равен $N$. Если пристроить к торцу такого цилиндра еще один такой же с потоком $N$, получим удвоение $L$ и удвоение $\Delta T$, а если пристроить сбоку, то получим удвоение $N$ и $S$ (рис.1). Таким образом, $N$ оказывается пропорционально $S \Delta T / L$, или

$$
N=\frac{\lambda \Delta T S}{L},
$$

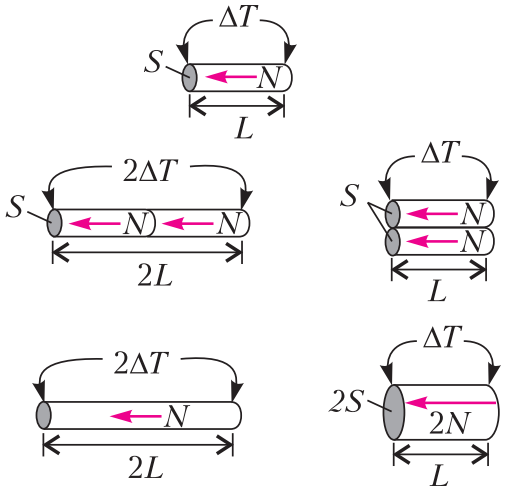

Pис. 1

где $\lambda$ - коэффициент пропорциональности, который как раз и называют теплопроводностью.

Определим размерность коэффициента теплопроводности и подумаем, какое предположение мы не сделали, а без него наша формула не то чтобы совсем ошибочна, но на экзамене придраться можно. Размерность $\lambda$ есть Вт/(м·К), а предположений по меньшей мере три - одно серьезное и два не очень. Не серьезные, что и $S$ и $L-$ константы, не зависящие от $\Delta T$. В данном случае это действительно так, потому что при нагреве на 300 К они изменяются обычно не более чем на $0,3 \%$. Это не означает, правда, что тепловыми расширениями можно пренебрегать в других случаях! А серьезное предположение, что $\lambda-$ константа. Для многих материалов это не так. При нагреве на $300 \mathrm{~K}$ теплопроводность может и увеличиться на треть и уменьшиться на $30 \%$, а это обычно существенно. Складывая два цилиндра торцами, мы не только увеличили вдвое $\Delta T$, но и увеличили сами значения $T$, а на возможную зависимость $\lambda(T)$ внимания не обратили. Но для нашей конкретной задачи это не важно - очень большие нагревы мы, понятное дело, не рассматриваем. Формулу $N=\frac{\lambda \Delta T S}{L}$ полезно запомнить. Хоть мы ее вывели для простейшей (одномерной) задачи, но она бывает полезна всегда, когда теплообмен «вбок» мал. Ведь при использовании теплоизоляции (одежда, строительство) это бывает чаще всего.

Теперь вернемся к нашей задаче. Пусть у нас есть полупространство, в нашем случае рука человека (опять, естественно, одномер- 


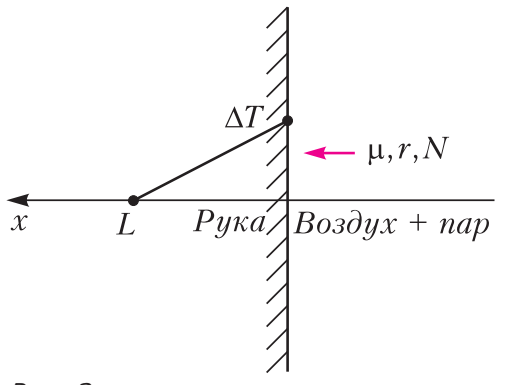

Рис. 2

ная задача), на границу которого падает тепловой поток $N$, приходящийся на площадь $S$ (рис.2). У нас этот поток создается конденсацией пара, на площадь $S$ падает поток вещества $\mu$ (размерность кг/с) с теплотой фазового перехода $r$ (размерность Дж/кг). Настоящим решением была бы функция $\Delta T(x, t)$, говорящая, на сколько нагреется вещество на глубине $x$ за время $t$. Получение решения в таком виде требует решения уравнения в частных производных, а мы этого не умеем. Поэтому рассмотрим самое сложное приближение, с которым мы справимся математически, и оно же самое простое, позволяющее получить разумный ответ. Совпадение этих двух приближений принципиально важный момент. В физике это случается далеко не всегда, но в данном случае нам повезло.

Будем считать, что температура падает в глубину линейно. Запишем очевидные уравнения:

$N=\mu r, Q=N t, Q=\frac{c m \Delta T}{2}, m=\rho S L, N=\frac{\lambda \Delta T S}{L}$,

где $Q$ - количество теплоты, $m$ - масса, $\rho$ плотность. Во втором уравнении для $Q$ произведено деление на 2, потому что мы использовали модель с линейной и непрерывной функцией $\Delta T(x)$. Менее реалистичной была бы модель $\Delta T(x)=$ const при $0<x<L$, в этом случае исчезло бы деление на два. Ну вот, решаем эту систему и получаем

$$
\Delta T(t)=\left(\frac{\mu r}{S}\right)\left(\frac{2 t}{c \rho \lambda}\right)^{1 / 2} .
$$

Прежде чем радостно подставлять числа, надо проверить размерность и разумность результата. Размерность проверьте сами, а разумностью займемся вместе.
То, что впереди стоит $(\mu r / S)$, - это правильно, поскольку это просто плотность мощности. То, что она в первой степени следствие «линейности задачи», постоянства $\lambda$. Энергии и мощности действительно суммируются (кстати, если они ноль, то нет никакого нагрева). Дальше - интереснее, время получилось под корнем. Так как энергия, введенная в систему, т.е. $Q$, растет со временем линейно, это значит, что как $t^{1 / 2}$ растет не только $\Delta T$, но и $L$. Да, из наших уравнений получаем

$$
L=\left(\frac{2 \lambda t}{c \rho}\right)^{1 / 2} .
$$

Эту формулу полезно запомнить, она позволяет оценить, на какое расстояние распространится в среде тепло за какое-то время, если мы знаем параметры среды.

Теперь займемся цифрами. Возьмем $r=$ $=2,2$ МДж/кг, $S=1 \mathrm{~cm}^{2}, t=0,2 \mathrm{c}, \rho=10^{3} \mathrm{\kappa г} / \mathrm{m}^{3}$, $c=3000$ Дж $/(\kappa г \cdot K), \lambda=0,5$ Вт $/($ м $\cdot$ K $)$. Две последние цифры для мяса находим в интернете, остальное очевидно. Но у нас проблема с величиной $\mu-$ как можно было бы ее оценить? Есть три варианта - экспериментальный, смешанный и теоретический. Первый: на секунду внести в поток пара из носика чайника блюдце или чашку и посмотреть, сколько воды на них конденсируется. Второй вариант: посмотреть, за какое время выкипает некоторое количество воды, и пересчитать это в поток вещества. Третий: просто вычислить $\mu$, исходя из мощности чайника. Первый вариант имеет в бытовых условиях низкую точность, третий плох тем, что мы не знаем КПД чайника. Однако в общем все три способа дают примерно одинаковые ответы - от $10^{-4}$ кг/с до $3 \cdot 10^{-4}$ кг/ с.

Но не вздумайте прямо подставлять! Это же поток на самом носике, а реально ближе 10 см от носика руку никто не проносит. Тогда надо сбросить еще полтора порядка учесть квадрат отношения этого расстояния к диаметру отверстия в носике 2 см. (Ибо мы живем в трехмерном пространстве - вы же помните про квадрат расстояния в законе всемирного тяготения и в законе Кулона?)

В итоге получается для перегрева $\Delta T$ разумная цифра - 60 К. И это за 0,2 секунды, так что будьте осторожны! 